\title{
Appressorium Formation and Tomato Fruit Infection by Colletotrichum coccodes
}

\author{
S. Sanogo, Former Graduate Research Assistant, R. E. Stevenson, Senior Research Assistant, and S. P. Penny- \\ packer, Emeritus Professor, Department of Plant Pathology, The Pennsylvania State University, University Park \\ 16802
}

\begin{abstract}
Sanogo, S., Stevenson, R. E., and Pennypacker, S. P. 2003. Appressorium formation and tomato fruit infection by Colletotrichum coccodes. Plant Dis. 87:336-340.

Tomato fruit grown for commercial processing are harvested when the majority of the fruit are at the full, red-ripe stage of development. At this physiological stage, marketable yields often are reduced significantly by Colletotrichum coccodes. Appressorium formation and the infection of tomato fruit by $C$. coccodes were investigated in controlled-environment experiments. Conidia of $C$. coccodes were subjected to five temperature treatments $\left(10\right.$ to $34^{\circ} \mathrm{C}$ with $6^{\circ} \mathrm{C}$ increments), and eight incubation periods ( 3 to $24 \mathrm{~h}$ with 3 -h increments). The highest proportion of conidia that formed appressoria occurred at 16 and $22^{\circ} \mathrm{C}$. Appressoria were formed within as few as $6 \mathrm{~h}$ of incubation at 16,22 , and $28^{\circ} \mathrm{C}$. In contrast, incubation periods of at least 15 and $18 \mathrm{~h}$ were required for appressoria to form at 34 and $10^{\circ} \mathrm{C}$, respectively. Appressorium formation was significantly reduced by 0.1 to $0.2 \mathrm{ppm}$ of the fungicide chlorothalonil, and no appressoria formed at concentrations $>0.4 \mathrm{ppm}$. When tomato fruit were inoculated with $C$. coccodes at three inoculum densities $\left(2 \times 10^{5}, 6 \times 10^{5}\right.$, and $10 \times 10^{5}$ conidia/ml $)$ and incubated in dew chambers for 8,16 , and $24 \mathrm{~h}$ at $5^{\circ} \mathrm{C}$ increments from 15 to $35^{\circ} \mathrm{C}$, there was no significant interaction among inoculum density, dew period, and temperature. In general, across all inoculum densities and dew periods, anthracnose severity levels were greater for each $5^{\circ} \mathrm{C}$ increase in temperature from $15^{\circ} \mathrm{C}$ until its maximum level was observed at $30^{\circ} \mathrm{C}$. However, when the fruit were exposed to $35^{\circ} \mathrm{C}$, disease development was minimal. At temperatures from 15 to $30^{\circ} \mathrm{C}$ anthracnose severity increased proportionally as dew-period duration and inoculum density increased.
\end{abstract}

The management of Colletotrichum coccodes (Wallr.) S. J. Hughes, the causal agent of anthracnose on processing tomato (Lycopersicon esculentum Mill.), relies heavily on the application of protectant fungicides such as chlorothalonil (tetrachloroisophthalonitrile). A current trend in the chemical control of foliar and fruit diseases of tomato is toward the design and implementation of disease-warning systems that optimize the timing of applications needed to provide adequate control of the pathogen. Although systems such as FAST (13) and TOM-CAST (16), that were designed for the control of early

Corresponding author: S. Sanogo

E-mail: ssanogo@nmsu.edu

Current address of S. Sanogo: N141 Skeen Hall, MSC 3BE, Las Cruces, NM 88003

Financial support for this research was provided by the Pennsylvania Agricultural Experiment Station and The Pennsylvania Department of Agriculture, Agricultural Research Program Contract ME445110. This is a portion of S. Sanogo's Ph.D. dissertation submitted to The Pennsylvania State University. Contribution 2054, Department of Plant Pathology, The Pennsylvania Agricultural Experiment Station.

Accepted for publication 22 October 2002.

Publication no. D-2003-0203-01R

(C) 2003 The American Phytopathological Society blight, are reported to aid in the control of tomato anthracnose (10), they do not incorporate epidemiological information on C. coccodes. The occurrence of significant yield reductions due to anthracnose during growing seasons when early blight is controlled is attributed to the absence of models derived from the biological response of C. coccodes to local weather conditions. Therefore, the results of supplementary studies on various facets of the infection cycle of $C$. coccodes are needed to improve the capability of currently used forecasters, or to design new systems to accurately manage tomato anthracnose epidemics. The need for research in this area has been indicated in other reports $(3,10)$.

A step of paramount importance in the infection process of many fungi is the formation of appressoria that facilitate both attachment of the germ tube onto plant surfaces and penetration of the host (1). Dillard (5) examined the effect of temperature and fungicides on conidial germination of $C$. coccodes, but the effects of these factors on appressorium formation were not reported. In at least two studies, appressorium formation was examined in relation to temperature (11) and incubation period (2). Combined effects of temperature and incubation period on formation of appressoria have not, however, been established quantitatively for $C$. coccodes.

Infection of fruit and other plant parts by C. coccodes hinges upon factors such as temperature, moisture, host maturity, and inoculum density. Fulton (9) studied the effect of fruit age on infection and lesion development, but did not define the moisture and temperature conditions under which his experiment was conducted. Under clearly defined experimental conditions, Dillard (6) separately examined the effects of moisture, temperature, and inoculum density on infection and lesion development of $C$. coccodes on ripe fruit. However, the combined effect of these factors was not investigated.

This study examined the effects of (i) temperature, incubation period, and chlorothalonil on appressorium formation; and (ii) temperature, inoculum density, and dew period on fruit infection by $C$. coccodes.

\section{MATERIALS AND METHODS}

Maintenance of cultures and production of conidia. The isolate of $C$. coccodes used in the studies described herein was obtained from naturally infected tomato fruit harvested from a research field plot at the Russell E. Larson Agricultural Research Center, Rock Springs, PA $\left(40^{\circ} 42^{\prime} 53^{\prime \prime} \mathrm{N}, 77^{\circ} 56^{\prime} 25^{\prime \prime} \mathrm{W}\right)$. Pathogenicity was maintained by inoculating ripe tomato fruit with this isolate every 6 to 8 weeks. Tissue from the inoculated fruit was then plated on potato dextrose agar (PDA) acidified with $1 \mathrm{ml}$ of $25 \%$ lactic acid/liter. For conidia production, PDA medium contained in $9-\mathrm{cm}$ plastic petri plates was seeded with 5-mm-diameter agar discs from a 7-day-old culture of $C$. coccodes. The plates then were incubated for 7 to 10 days in a temperature-controlled chamber (Model RI-50-555-A, Revco, Rheem Manufacturing Co., Asheville, NC) at $27^{\circ} \mathrm{C}$ under continuous light provided by cool-white fluorescent lamps with an intensity of approximately $440 \mu \mathrm{W} / \mathrm{cm}^{2}$ (17). Conidia were harvested by flooding the plates with sterile distilled water and gently scraping the surface of the medium with a camel-hair brush to dislodge the conidia. The number of conidia per milliliter of suspension was assessed with a hemacytometer.

Effect of temperature and incubation period on appressorium formation. The effects of five temperatures and eight incubation periods on formation of appressoria were evaluated in a factorial experiment using a procedure modified from Kubo (12). Two 10-by-10-mm pieces of DuPont 193 PUD-0, nonwaterproof cellophane (E. 
I. DuPont de Nemours, Clinton, IA), placed on $2 \%$ water agar medium in $5-\mathrm{cm}$ glass petri plates, were saturated with $2 \mathrm{ml}$ of $C$. coccodes conidial suspension ( 5 by 105 conidia/ml). Two plates were assigned to each temperature-incubation period combination. The plates were sealed with parafilm and placed in darkness in incubators (Model RI-50-555-A, Revco, Rheem Manufacturing Co., Asheville, NC) set at $10,16,22,28$, and $34^{\circ} \mathrm{C}$. Following $3,6,9$, $12,15,18,21$, and $24 \mathrm{~h}$ at each temperature, two plates were removed from each incubator, and the cellophane pieces were mounted in lacto-fuchsin to enhance microscopic observations. Appressorium formation was assessed on the first 100 conidia observed on each cellophane piece. The study was performed as a two-factor experiment in a split plot arranged in randomized complete block design with two replications. Temperature and incubation period were the whole plot and subplot factors, respectively. The complete experiment was performed two times.

Effect of chlorothalonil on appressorium formation. A cellophane bioassay procedure $(14,20)$ was used to establish chlorothalonil dosage levels required to inhibit appressorium formation by $C$. coccodes. The Bravo 500 and Bravo 720 (Zeneca, Inc., Wilmington, DE) formulations of this broad spectrum fungicide included 40.4 and $54.0 \%$, respectively, of the active ingredient (a.i) tetrachloroisophthalonitrile. Filter paper discs (18mm-diameter, Whatman No. 5) were saturated with fungicide solution at concentrations of $0.01,0.1,0.2,0.4,0.8$, or $1.0 \mathrm{ppm}$ (a.i.) or with distilled, deionized water. The saturated discs were then placed in individual wells of Coors U.S.A. (VWR Scientific Products, Philadelphia, PA) white porcelain spot plates. Each plate (118 by $155 \mathrm{~mm}$ ) contained 12 8-mm-deep depressions. Three cellophane discs (5-mmdiameter) were placed on each of the filter paper discs and a $0.5-\mu$ drop of a conidial suspension $\left(5 \times 10^{5}\right.$ conidia $\left./ \mathrm{ml}\right)$ was placed in the center of each cellophane disc. The spot plates were stacked on a porcelain support in a glass desiccator containing distilled water in the bottom to maintain high humidity during incubation. The humidity chamber was placed in darkness in an incubator and maintained at $22^{\circ} \mathrm{C}$ for 24 h. Then, the cellophane discs were mounted in a drop of lactophenol cottonblue for microscopic observations. Appressorium formation was based on the first 100 conidia observed on each cellophane disc. Inhibition of appressorium formation was assessed as $I(\%)=(1-F / C) \times 100$, where $F$ and $C$ represent percentages of conidia that produced appressoria on fungicide-treated and control (no fungicide) cellophane discs, respectively. Conidia response to chlorothalonil was evaluated from the results of three replications, with a replication consisting of three cellophane discs exposed to the distilled, deionized water control and each of the six fungicide concentrations. The entire experiment was performed four times.

Effect of temperature, inoculum density, and dew period on fruit infection. The influence of five temperature treatments, three inoculum densities, and three dew periods on fruit infection by $C$. coccodes was investigated. Two mature, disease-free red fruit ( 3 to $4 \mathrm{~cm}$ in diameter), harvested from greenhouse-grown tomato plants (cultivar Brigade), were assigned to each treatment combination. The fruit were sprayed until runoff with inoculum suspensions of $2 \times 10^{5}, 6 \times 10^{5}$, and $10 \times 10^{5}$ conidia $/ \mathrm{ml}$. The fruit were air dried on a laboratory bench and placed in dew chambers (Model E-54U-DL, Percival Mfg. Co., Boone, IA) set at $15,20,25,30$, and $35^{\circ} \mathrm{C}$. Following 8, 16, and $24 \mathrm{~h}$ of exposure to dew, two fruit were removed from each chamber and wiped with $95 \%$ alcohol. Sixteen 1-cm-diameter discs, approximately 1 to $2 \mathrm{~mm}$ thick, were arbitrarily sampled from the outermost layer of each fruit using a sterile cork borer. The discs were plated, epidermis down, onto acidified PDA medium in $10-\mathrm{cm}$ glass petri plates (4 discs per plate) and incubated in darkness at $22^{\circ} \mathrm{C}$ for 7 to 10 days. The number of discs yielding $C$. coccodes was recorded. The 5-by-3-by-3 experiment was conducted in a split-split plot design with temperature as whole plots, inoculum density as subplots, and dew period as subsubplots. The experiment was performed two times.

Data analysis. Analysis of variance and regression procedures were used to analyze the data associated with variations in appressorium formation that may be attrib- uted to conidia exposure to various concentrations of chlorothalonil, and the data which quantified the influences that several selected environmental parameters had on appressorium formation and fruit infection by $C$. coccodes. Transformations applied to the data sets included (i) the arcsine square-root transformation of the values representing the proportions of conidia that formed appressoria, (ii) the logarithm (base e) transformation of both the proportions of infected discs and the inoculum densities associated with the fruit infection study, and (iii) the logarithm (base e) transformation of the percentages of conidia that did not form appressoria due to the chlorothalonil treatments. Criteria for selecting the best statistical models included the examination of $R^{2}$ values, goodness-offit between expected and observed values, and residual plots.

\section{RESULTS}

Effect of temperature and incubation period on appressorium formation. Conidia of $C$. coccodes formed appressoria at all temperatures from 10 to $34^{\circ} \mathrm{C}$. The highest proportions of conidia that formed appressoria were observed at 16 and $22^{\circ} \mathrm{C}$ (Fig. 1). A minimum of $6 \mathrm{~h}$ of incubation was required for conidia to form appressoria at 16,22 , and $28^{\circ} \mathrm{C}$. At least $18 \mathrm{~h}$ of incubation was required for appressorium to form at 10 and $34^{\circ} \mathrm{C}$. The proportion of conidia that formed appressoria increased with increasing periods of incubation and was highest across all temperature treatments at $24 \mathrm{~h}$. No appressoria formed within $3 \mathrm{~h}$ when the conidia were subjected to any of the five treatment temperatures; therefore, the data for the 3-h incubation period were excluded from the data set

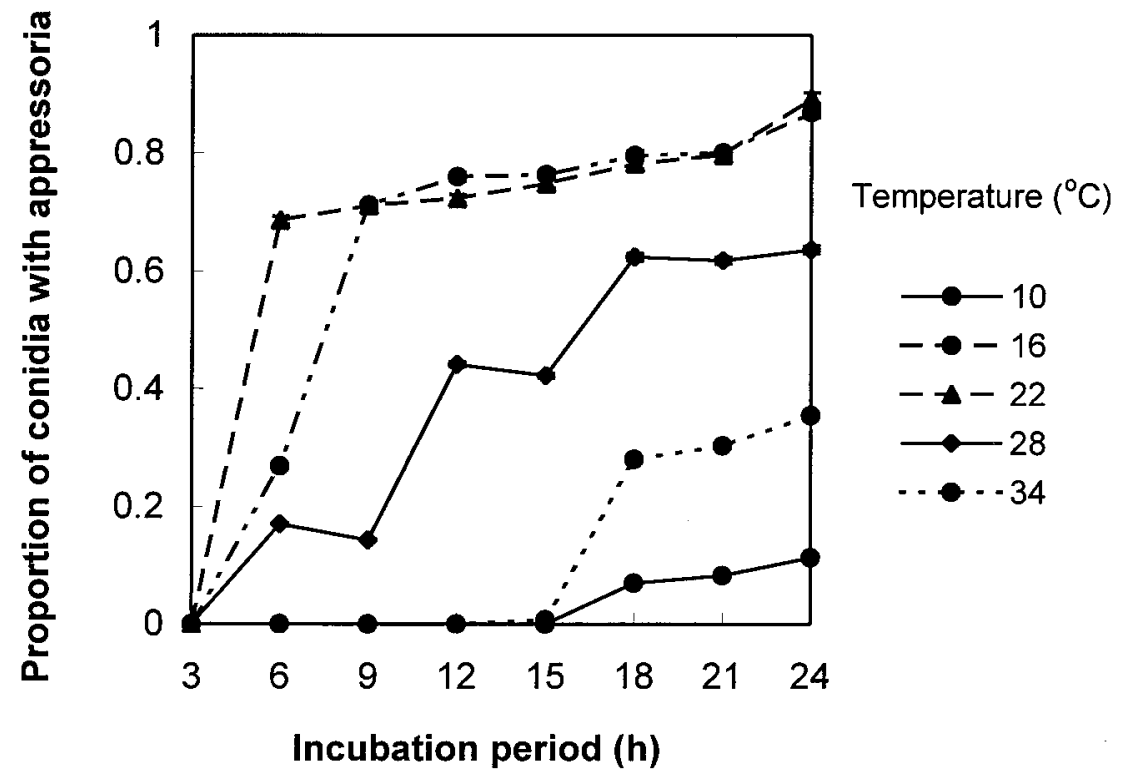

Fig. 1. Proportion of conidia of Colletotrichum coccodes producing appressoria in relation to five temperature treatments and eight incubation periods. Data points are means of two replicates of an experiment. Bars on data points are standard errors of the mean. Bars that are not visible have length less than the diameter of the data points. 
used in modeling this response. The full and reduced model approach (15) indicated there was no significant statistical difference between experimental replicates and the error variances were homogeneous. Analysis of variance indicated there were significant linear and quadratic relationships between the proportion of conidia with appressoria (arcsine square-root transformed) and temperature and incubation period. Using a stepwise regression procedure, the best fitting model was of the form: $Y=b_{0}+b_{1} T+b_{2} t+b_{3} T^{2}+b_{4} t T^{2}$, where $Y$ represents the arcsine-square roottransformed proportion of conidia that formed appressoria expressed in degrees, $T$ is temperature $\left({ }^{\circ} \mathrm{C}\right)$, and $t$ is the incubation period (h). The $R^{2}$ and $R a^{2}$ values for the chosen model were 0.89 and 0.88 , respectively, and all regression coefficients (Table 1) were significant $(0.0001<P<$ 0.014).

Effect of chlorothalonil on appressorium formation. Differences in the proportions of conidia of $C$. coccodes that formed appressoria were detected over each treatment concentration of chlorothalonil from 0.01 to $0.2 \mathrm{ppm}$. Within this span of treatments, inhibition of appressoria formation increased from approximately 17.0 to $96.6 \%$ and from 0.01 to $84.0 \%$, respectively, with increasing concentrations of the Bravo 500 and Bravo 720 formulations. No appressoria formed when the conidia were exposed to either formulation at concentrations $>0.4 \mathrm{ppm}$ (Fig. 2). For each formulation of chlorothalonil, regression analysis indicated there were no significant differences among the four replications of the study and there was a significant relationship between the natural logarithm (percent inhibition of appressorium formation), $Y$, and the inverse of the concentration of chlorothalonil (ppm), 1/ppm. The best fitting model was of the form: $Y=b_{0}$ $+b_{1}(1 / \mathrm{ppm})$. The estimated parameters for the chosen model are presented in Table 2.

Effect of temperature, inoculum density, and dew period on fruit infection. In general, across all inoculum densities and dew periods, anthracnose severity

Table 1. Parameter estimates for the equation relating appressorium formation by Colletotrichum coccodes to temperature and incubation period

\begin{tabular}{lcc}
\hline Parameters $^{\mathbf{a}}$ & Estimate & $\begin{array}{c}\text { Standard } \\
\text { error }\end{array}$ \\
\hline$b_{0}$ & -2.111 & 0.1560 \\
$b_{1}$ & 0.268 & 0.0134 \\
$b_{2}$ & 0.016 & 0.0054 \\
$b_{3}$ & -0.0006 & 0.00032 \\
$b_{4}$ & 0.00002 & 0.000008 \\
\hline
\end{tabular}

a The model was of the form $Y=b_{0}+b_{1} T+b_{2}$ $t+b_{3} T^{2}+b_{4} t T^{2}$, in which $Y, T$, and $t$ were arcsine-square root-transformed $\left(\sin ^{-1} Y^{1 / 2}\right)$ proportion of conidia with appressoria expressed in degrees, temperature $\left({ }^{\circ} \mathrm{C}\right)$ from 10 to 34 , and incubation period (h) from 6 to 24, respectively. levels increased with each $5^{\circ} \mathrm{C}$ increase in temperature from $15^{\circ} \mathrm{C}$ until its maximum level was observed at $30^{\circ} \mathrm{C}$ (Fig. 3). However, disease development was minimal when at $35^{\circ} \mathrm{C}$. Between 15 and $30^{\circ} \mathrm{C}$, anthracnose severity increased proportionally as dew-period duration and inoculum density increased. Analysis of variance indicated that there was no statistically significant three-factor interaction among temperature, inoculum density, and dew period $(P=0.1599)$. The best fitting model was $Y=b_{0}+b_{1} T+b_{2} I D+b_{3} t+b_{4} T^{2}$, in which $Y, T, I D$, and $t$ were the natural logarithm (base e) transformed proportions of infected discs, temperature $\left({ }^{\circ} \mathrm{C}\right)$, natural logarithm (base e) transformed inoculum densities, and dew period (h), respectively. Parameters from this model are presented in Table 3. All regression coefficients were significant $(P=0.0001)$ and the values of $R^{2}$ and $R a^{2}$ were 0.82 and 0.81 , respectively.

\section{DISCUSSION}

The present study has established a relationship between appressorium formation by $C$. coccodes and two important physical factors, temperature and moisture (incubation in presence of free moisture). This quantitative relationship embodies two characteristics of appressorium formation that are depicted by a quadratic trend with respect to temperature and a linear trend with respect to incubation period. Using cellophane membranes, we showed that regardless of temperature, the percentage of conidia with appressoria increased linearly with incubation period, reaching a maximum at about $24 \mathrm{~h}$. Byrne et al. (2) found a similar trend for appressorial formation on foliage of tomato plants maintained at $25^{\circ} \mathrm{C}$. Thus, although cellophane membranes provided an in vitro assessment of appressorial formation independent of interactions with the host plant, the results obtained using these membranes are comparable to those reported on host plant tissue.

Appressorium formation appeared to be favored by relatively low temperatures (16 to $22^{\circ} \mathrm{C}$ ). The rapid formation of appressoria at these temperatures might indicate the ability of $C$. coccodes to exploit conditions during nights with low temperature, prolonged dew period, and high relative humidity that are characteristic of many tomato fields during the summer growing season in north-central and northeastern regions of the United States. The optimum

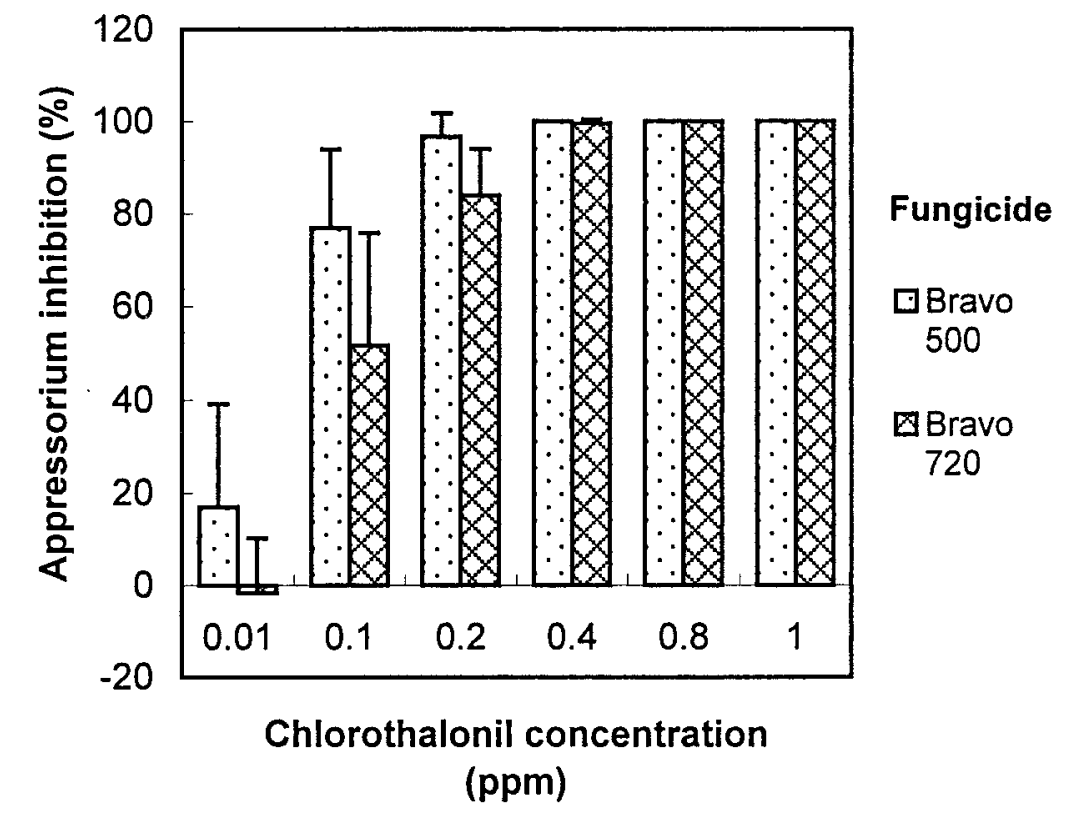

Fig. 2. Percent inhibition of appressorium formation by Colletotrichum coccodes at six concentrations of chlorothalonil. Data columns are means of four replicates of an experiment. Bars on columns are standard deviations.

Table 2. Parameter estimates for the equation relating appressorium formation by Colletotrichum coccodes to chlorothalonil concentrations

\begin{tabular}{lccccc}
\hline & \multicolumn{2}{c}{ Parameter estimates $^{\mathbf{a}}$} & & & \\
\cline { 2 - 4 } Formulation & $\mathbf{b}_{\mathbf{0}}$ & $\mathbf{b}_{\mathbf{1}}$ & $\boldsymbol{P}(>\boldsymbol{F})$ & $\boldsymbol{R}^{\mathbf{2}}$ & $\boldsymbol{R}^{\mathbf{a}} \boldsymbol{~}$ \\
\hline Bravo 500 & $4.5874(0.0498)$ & $-0.0116(0.0016)$ & $<0.0001$ & 71.5 & 70.1 \\
Bravo 720 & $4.5235(0.0928)$ & $-0.0279(0.0030)$ & $<0.0001$ & 80.8 & 79.8 \\
\hline
\end{tabular}

a The model was of the form: $\operatorname{Ln}(\%$ Inhibition $)=b_{0}+b_{1}(1 / p p m)$, in which treatment concentrations ranged from 0.01 to $1.0 \mathrm{ppm}$. Numbers in parentheses are the standard deviations of the estimated parameters. 
temperature value of $22^{\circ} \mathrm{C}$ for appressoria formation has also been reported as optimum for conidial germination (5).

In addition to the physical environment, this study shows that the chemical environment also exerts a significant effect on appressorium formation by $C$. coccodes, as demonstrated by the significant reduction and inhibition of appressoria formation by chlorothalonil. Chlorothalonil was included in this study because it is the most widely used broad-spectrum fungicide for controlling anthracnose in the United States (7), and it plays a vital role in the
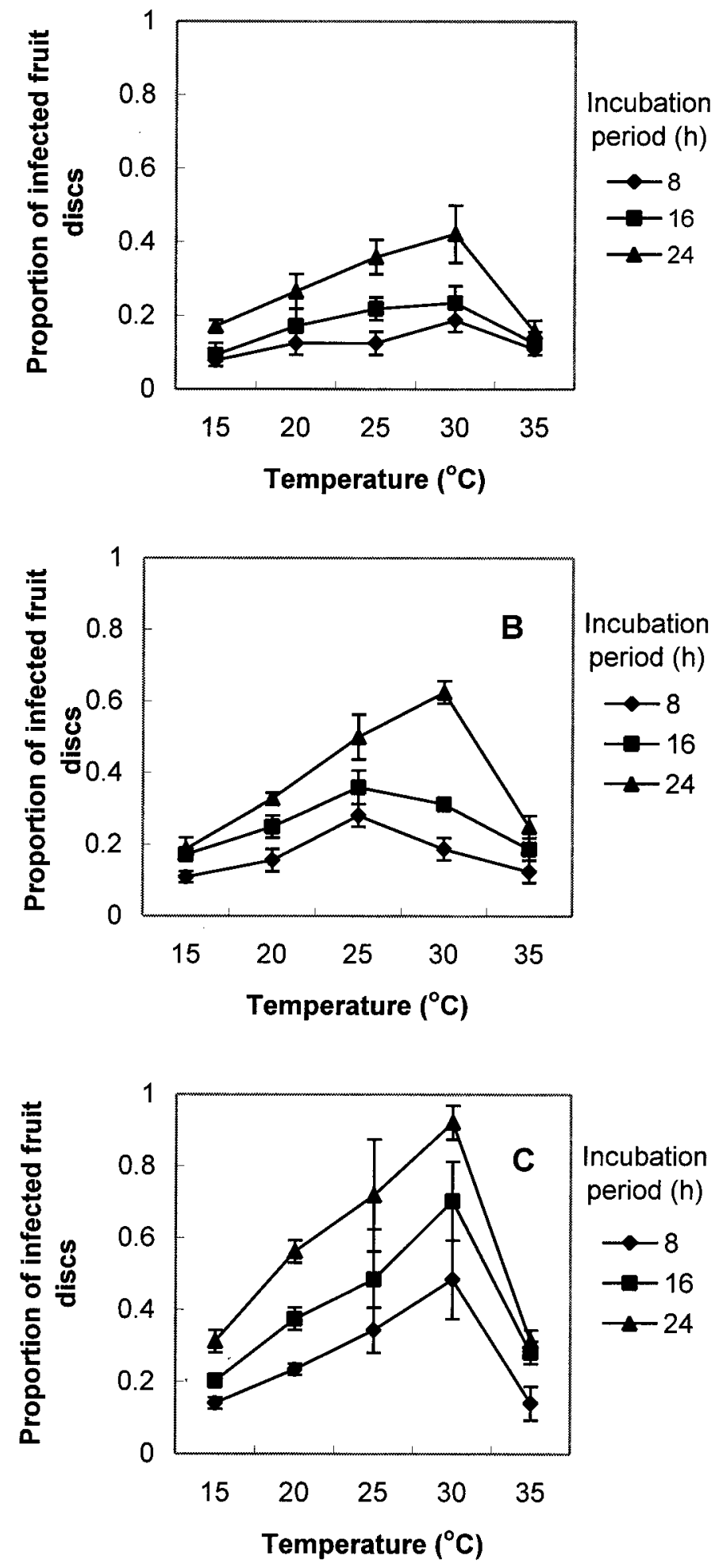

Fig. 3. Proportion of tomato fruit discs infected by Colletotrichum coccodes at five temperature treatments and three dew periods when fruit were inoculated at three inoculum densities of $\mathbf{A}, 2 \times$ $10^{5}, \mathbf{B}, 6 \times 10^{5}$, and $\mathbf{C}, 10 \times 10^{5}$ conidia/ml. Data points are means of two replicates of an experiment. Bars on data points are standard errors of the mean. Bars that are not visible have length less than the diameter of the data points.

feasibility of commercial production of processing tomato in the Midwest (3). Our assessment of this fungicide in relation to appressoria formation by $C$. coccodes agrees with the results obtained on conidial germination in previous work (5). Specifically, we found that inhibition of appressoria formation was about 20 to over $90 \%$ from 0.01 to $0.2 \mathrm{ppm}$, and no appressoria formed when the conidia were exposed to concentrations above $0.4 \mathrm{ppm}$. In the previous study, conidial germination was 7 to $10 \%$ of control at $0.1 \mathrm{ppm}$, and completely inhibited at $1.0 \mathrm{ppm}$ (5). The results of our studies suggest that a minimum of $0.2 \mathrm{ppm}$ of chlorothalonil may be needed to prevent appressoria formation and, therefore, minimize fruit infection. This finding may be related to a study conducted by Freeborn (8) who reported that fruit rot due to anthracnose increased and marketable tomato fruit yields were reduced when residual concentrations of chlorothalonil on fruit surfaces approached $0.2 \mu \mathrm{g} / \mathrm{cm}^{2}$.

We developed a quantitative relationship of temperature, dew period, and inoculum density to fruit infection using regression methods. The range of temperatures examined was consistent with that normally encountered during the summer growing season in Pennsylvania. The levels of inoculum density selected were within the realm of previous work (6) to allow comparison of results. However, it is not known if our treatment levels were reflective of field inoculum densities. As far as we are aware, levels of $C$. coccodes conidia that may be found on tomato fruit grown under natural field conditions have not been reported. We found that, over all temperatures from 15 to $30^{\circ} \mathrm{C}$, observed levels of anthracnose severity were greater both at each increased period of dew and with each increased level of inoculum density. These results congrue with those obtained by Dillard (6). Except at $15^{\circ} \mathrm{C}$, our results also compare well with those obtained by Byrne et al. (4), who examined the relationship of dew period and temperature to infection of tomato foliage by

Table 3. Parameter estimates for the equation relating tomato fruit infection by Colletotrichum coccodes to temperature, inoculum density, and dew period

\begin{tabular}{lcc}
\hline Parameters $^{\mathbf{a}}$ & Estimate & $\begin{array}{c}\text { Standard } \\
\text { error }\end{array}$ \\
\hline$b_{0}$ & -13 & 0.685 \\
$b_{1}$ & 0.413 & 0.0340 \\
$b_{2}$ & 0.446 & 0.0420 \\
$b_{3}$ & 0.048 & 0.0042 \\
$b_{4}$ & -0.008 & 0.0006 \\
\hline
\end{tabular}

a The equation was of the form $Y=b_{0}+b_{1} T+$ $b_{2} I D+b_{3} t+b_{4} T^{2}$, in which $Y, T, I D$, and $t$ were the natural logarithm (base e) transformed proportions of infected discs, temperature $\left({ }^{\circ} \mathrm{C}\right)$ from 15 to 35 , natural logarithm (base e) transformed inoculum densities from $2 \times 10^{5}$ to $10 \times 10^{5}$, and dew period (h) from 8 to 24 , respectively. 
C. coccodes. Fruit infection occurred in this study at $15^{\circ} \mathrm{C}$ whereas, at this temperature, Byrne et al. (4) did not observe any foliar infection. Perhaps this discrepancy may be ascribed to factors such as age and susceptibility difference in experimental materials, inoculum density, and microenvironment variations.

This work was conducted on the assumption that free moisture or high humidity conditions persist over a 24-h period following deposition of conidia on tomato fruit or foliage. However, under field conditions, free moisture or high relative humidity periods often are interrupted by dry periods. Investigation into the effects of interrupted free moisture or high relative humidity on appressorial formation and fruit infection should yield useful adjustments to the results reported in this study.

The present study provides additional information supporting a previous report (19) in which we examined the relationship of tomato anthracnose to rainfall, dew, and solar radiation for three intervals of temperature (16 to 28,20 to 30 , and 15 to $30^{\circ} \mathrm{C}$ ) when relative humidity equaled or exceeded $90 \%$. The temperature intervals of 16 to 28 and 20 to $30^{\circ} \mathrm{C}$ were indicated as optimal for appressoria formation and fruit infection, respectively, under controlled environment. The interval of 15 to $30^{\circ} \mathrm{C}$ was previously shown to be optimal for sporogenic germination (18). Results from this work could be used in establishing empirical decision rules for determining when fruit infection by $C$. coccodes is likely. For example, at temperatures from 16 to $28^{\circ} \mathrm{C}$, fruit infection would not be expected at wetness periods of less than $6 \mathrm{~h}$ duration.
Availability of such data should facilitate modifications of existing disease-warning systems or implementation of new ones.

\section{ACKNOWLEDGMENTS}

We thank J. L. Rosenberger, Head of the Department and Professor of Statistics at The Pennsylvania State University, for assistance with the study designs and data analysis; and M. L. Gleason, Professor of Plant Pathology at The Iowa State University, for his critical review of this manuscript.

\section{LITERATURE CITED}

1. Agrios, N., G. 1997. Plant Pathology. 4th ed. Academic Press, San Diego, CA.

2. Byrne, J. M., Hausbeck, M. K., and Hammerschmidt, R. 1997. Conidial germination and appressorial formation by Colletotrichum coccodes on tomato foliage. Plant Dis. 81:715718.

3. Byrne, J. M., Hausbeck, M. K., and Latin, R. X. 1997. Efficacy and economics of management strategies to control anthracnose fruit rot in processing tomatoes in the Midwest. Plant Dis. $81: 1167-1172$

4. Byrne, J. M., Hausbeck, M. K., Meloche, C., and Jarosz, A. M. 1998. Influence of dew period and temperature on foliar infection of greenhouse-grown tomato by Colletotrichum coccodes. Plant Dis. 82:639-641.

5. Dillard, H. R. 1988. Influence of temperature, $\mathrm{pH}$, osmotic potential, and fungicide sensitivity on germination of conidia and growth from sclerotia of Colletotrichum coccodes in vitro. Phytopathology 78:1357-1361.

6. Dillard, H. R. 1989. Effect of temperature, wetness duration, and inoculum density on infection and lesion development of Colletotrichum coccodes on tomato fruit. Phytopathology 79:1063-1066.

7. Dillard, H. R., Johnston, S. A., and Hamilton, G. H. 1997. An assessment of fungicide benefits for the control of fungal diseases of processing tomatoes in New York and New Jersey. Plant Dis. 81:677-681.

8. Freeborn, R. J. 1985. Residual levels of chlorothalonil on tomatoes. M.S. thesis. The
Pennsylvania State University, University Park.

9. Fulton, J. P. 1948. Infection of tomato fruits by Colletotrichum phomoides. Phytopathology 38:235-246

10. Gleason, M. L., MacNab, A. A., Pitblado, R. E., Ricker, M. D., East, D. A., and Latin, R. X 1995. Disease-warning systems for processing tomatoes in Eastern North America: Are we there yet? Plant Dis. 79:113-121.

11. Illman, W. I. 1960. Anthracnose disease of tomato. Ph.D. thesis. The University of Western Ontario, London, Canada.

12. Kubo, Y., Suzuki, K., Furusawa, I., Ishida, N., and Yamamoto, M. 1982. Relation of appressorium pigmentation and penetration of nitrocellulose membranes by Colletotrichum lagenarium. Phytopathology 72:498-501

13. Madden, L., Pennypacker, S. P., and MacNab, A. A. 1978. FAST, a forecast system for Alternaria solani on tomato. Phytopathology 68:1354-1358.

14. Neely, D. 1970. Persistence of foliar protective fungicides. Phytopathology 60:15831586.

15. Neter, J., and Wasserman, W. 1974. Applied Linear Statistical Models. Irwin, Inc., Homewood, IL.

16. Pitblado, R. E. 1992. Development of a weather-timed fungicide spray program for field tomatoes. (Abstr.) Can. J. Plant Pathol. 10:371.

17. Raid, R. N., and Pennypacker, S. P. 1987. Weeds as hosts for Colletotrichum coccodes. Plant Dis. 71:643-646.

18. Sanogo, S., and Pennypacker, S. P. 1997 Factors affecting sporogenic and myceliogenic germination of sclerotia of Colleto trichum coccodes. Plant Dis. 81:333-336.

19. Sanogo, S., Pennypacker, S. P., Stevenson, R. E., and MacNab, A. A. 1997. Weather variables associated with infection of tomato fruit by Colletotrichum coccodes. Plant Dis. 81:753-756.

20. Stevenson, R, Sanogo, S., and Pennypacker, S. P. 1994. Effect of two formulations of chlorothalonil on appressorial formation by Colletotrichum coccodes. (Abstr.) Phytopathology 84:1375. 